\title{
Ground state of two electrons on a sphere
}

\author{
Pierre-François Loos and Peter M. W. Gill* \\ Research School of Chemistry, Australian National University, Canberra, Australian Capital Territory 0200, Australia
}

(Received 12 May 2009; published 30 June 2009)

\begin{abstract}
We have performed a comprehensive study of the singlet ground state of two electrons on the surface of a sphere of radius $R$. We have used electronic structure models ranging from restricted and unrestricted HartreeFock theories to explicitly correlated treatments, the last of which leads to near-exact wave functions and energies for any value of $R$. Møller-Plesset energy corrections (up to fifth-order) are also considered, as well as the asymptotic solution in the large- $R$ regime.
\end{abstract}

DOI: 10.1103/PhysRevA.79.062517

PACS number(s): 31.15.ac, 31.15.ve, 31.15.xp, 31.15.xr

\section{INTRODUCTION}

Exactly (or very accurately) solvable models have ongoing value, and are valuable both for illuminating more complicated systems and for testing theoretical approaches, such as density-functional methods [1-3]. One such model is the Hooke's law atom (or Harmonium), which is composed of two electrons bound to a nucleus by a harmonic potential but repelling Coulombically. This system was first considered more than 40 years ago by Kestner and Sinanoglu [4] but solved analytically in 1989 by Kais et al. [5] for a particular value of the harmonic force constant and, later, for a countably infinite set of force constants [6].

A related system, studied by Alavi and co-workers [7-9], consists of two electrons, interacting through a Coulomb potential but confined within a ball of radius $R$. This possesses a number of interesting features, including the formation of a "Wigner molecule" for large $R$ (LR) [10]. The spontaneous formation of such molecules can also occur in quantum dots and is analogous to the Wigner crystallization [11] of the uniform electron gas.

If the two electrons are constrained to remain on the surface of the sphere, one obtains a model that Berry and coworkers have used [12-15] to understand both weakly and strongly correlated systems, such as the ground and excited states of the helium atom, and also to suggest the "alternating" version of Hund's rule [16]. Seidl studied this system in the context of density-functional theory [17] in order to test the interaction-strength interpolation model [18]. For this purpose, he derived accurate solutions in both the weakinteraction limit (the small- $R$ regime) and the stronginteraction limit (the large- $R$ regime). He also obtained accurate results by numerical integration of the Schrödinger equation.

In this paper, we are interested in the ${ }^{1} S$ ground state of two electrons on the surface of a sphere of radius $R$. This allows us to restrict our study to the symmetric spatial part of the wave function and ignore the spin coordinates. We have extended Seidl's analysis and performed an exhaustive study using a range of models. We restrict our analysis to the repulsive potential case; the strong-attraction limit (attractive potential) is carefully examined in Ref. [17].

\footnotetext{
*Corresponding author; peter.gill@anu.edu.au
}

Restricted and unrestricted Hartree-Fock (HF) solutions are discussed in Sec. III, and the strengths and weaknesses of Møller-Plesset (MP) perturbation theory [19] in Sec. IV. We consider asymptotic solutions for large $R$ in Sec. V and, in Sec. VI, we explore several variational schemes including explicitly correlated techniques [20-24] that enforce the cusp condition $[25,26]$. Atomic units are used throughout.

\section{HAMILTONIAN}

The absolute position of the $i$ th electron is defined by its spherical polar angles $\Omega_{i}=\left(\theta_{i}, \phi_{i}\right)$. The relative position of the electrons is conveniently measured by the interelectronic angle $\theta$, which they subtend at the origin. These coordinates are related by

$$
\cos \theta=\cos \theta_{1} \cos \theta_{2}+\sin \theta_{1} \sin \theta_{2} \cos \left(\phi_{1}-\phi_{2}\right),
$$

and we have $0 \leq u \equiv\left|\mathbf{r}_{1}-\mathbf{r}_{2}\right| \leq 2 R$.

The Hamiltonian is

$$
\hat{H}=\hat{T}+u^{-1},
$$

where

$$
\hat{T}=\hat{T}_{1}+\hat{T}_{2}=-\frac{\nabla_{1}^{2}}{2}-\frac{\nabla_{2}^{2}}{2}
$$

is the kinetic operator for both electrons and $u^{-1}$ is the Coulomb operator. In terms of $\theta$, the Hamiltonian is

$$
\hat{H}=-\frac{1}{R^{2}}\left(\frac{d^{2}}{d \theta^{2}}+\cot \theta \frac{d}{d \theta}\right)+\frac{1}{2 R} \csc \frac{\theta}{2},
$$

in which form it becomes clear that the kinetic and potential parts of $\hat{H}$ scale with $R^{-2}$ and $R^{-1}$, respectively.

\section{HARTREE-FOCK APPROXIMATIONS}

\section{A. Restricted Hartree-Fock}

In the HF approximation, each electron feels the mean field generated by the other electron [27]. The restricted Hartree-Fock (RHF) solution,

$$
\Phi^{\mathrm{RHF}}\left(\Omega_{1}, \Omega_{2}\right)=\Psi^{\mathrm{RHF}}\left(\Omega_{1}\right) \Psi^{\mathrm{RHF}}\left(\Omega_{2}\right),
$$

places both electrons in an orbital $\Psi^{\mathrm{RHF}}$ that is an eigenfunction of the Fock operator 


$$
\hat{F}_{1}=\hat{T}_{1}+\int \frac{\left|\Psi^{\mathrm{RHF}}\left(\Omega_{2}\right)\right|^{2}}{u} R^{2} d \Omega_{2},
$$

with $d \Omega_{2}=\sin \theta_{2} d \theta_{2} d \phi_{2}$.

By definition, the one-electron basis function

$$
\Psi_{\ell m}\left(\Omega_{i}\right)=\frac{Y_{\ell m}\left(\Omega_{i}\right)}{R},
$$

where $Y_{\ell m}$ is the spherical harmonic of degree $\ell$ and order $m$ is an eigenfunction of $\hat{T}_{i}$ with eigenvalue $\ell(\ell+1) /\left(2 R^{2}\right)$. Using the partial-wave expansion [28]

$$
u^{-1}=R^{-1} \sum_{\ell=0}^{\infty} P_{\ell}(\cos \theta)
$$

and the addition theorem [29]

$$
P_{\ell}(\cos \theta)=\frac{4 \pi}{2 \ell+1} \sum_{m=-\ell}^{+\ell} Y_{\ell m}^{*}\left(\Omega_{1}\right) Y_{\ell m}\left(\Omega_{2}\right),
$$

it is straightforward to show that

$$
\int \frac{\left|\Psi_{00}\left(\Omega_{2}\right)\right|^{2}}{u} R^{2} d \Omega_{2}=\frac{1}{R} .
$$

The orbital $\Psi_{00}\left(\Omega_{i}\right)$ is thus an eigenfunction of $\hat{F}_{i}$ with the eigenvalue $1 / R$. Moreover, it follows from the orthogonality of the spherical harmonics that

$$
\left\langle\Psi_{\ell m}\left(\Omega_{1}\right)\left|\hat{F}_{1}\right| \Psi_{00}\left(\Omega_{1}\right)\right\rangle=\delta_{\ell, 0} \delta_{m, 0},
$$

which ensures the stationarity of the RHF energy with respect to the orbitals $\Psi_{\ell m}$.

The ground-state RHF energy is thus

$$
E^{\mathrm{RHF}}=\frac{1}{R}
$$

and the normalized RHF wave function is

$$
\Phi^{\mathrm{RHF}}=\frac{1}{4 \pi R^{2}},
$$

which yields a uniform electron density over the surface of the sphere.

\section{B. Unrestricted Hartree-Fock}

When $R$ exceeds a critical value, a second unrestricted HF (UHF) solution develops [30-32] in which the two electrons tend to localize on opposite sides of the sphere. This is analogous to the UHF description of a dissociating $\mathrm{H}_{2}$ molecule [27].

To obtain this symmetry-broken solution

$$
\Phi^{\mathrm{UHF}}\left(\theta_{1}, \theta_{2}\right)=\Psi^{\mathrm{UHF}}\left(\theta_{1}\right) \Psi^{\mathrm{UHF}}\left(\pi-\theta_{2}\right),
$$

we expand the orbital as

$$
\Psi^{\mathrm{UHF}}\left(\theta_{i}\right)=\sum_{\ell=0}^{\infty} C_{\ell} \Psi_{\ell}\left(\theta_{i}\right)
$$

where the $\Psi_{\ell}\left(\theta_{i}\right)=Y_{\ell}\left(\theta_{i}\right) / R=Y_{\ell 0}\left(\Omega_{i}\right) / R$ are zonal spherical harmonics. The Fock matrix elements in this basis are

$$
\begin{aligned}
F_{\ell_{1} \ell_{2}} & =\left\langle\Psi_{\ell_{1}}\left(\theta_{i}\right)\left|\hat{F}_{i}\right| \Psi_{\ell_{2}}\left(\theta_{i}\right)\right\rangle \\
& =\frac{\ell_{1}\left(\ell_{1}+1\right)}{2 R^{2}} \delta_{\ell_{1}, \ell_{2}}+\sum_{\ell_{3}, \ell_{4}=0}^{\infty} C_{\ell_{3}} C_{\ell_{4}} G_{\ell_{1} \ell_{2} \ell_{4}},
\end{aligned}
$$

where the two-electron integrals are

$$
G_{\ell_{1} \ell_{2}}^{\ell_{3} \ell_{4}}=\left\langle\Psi_{\ell_{1}}\left(\theta_{1}\right) \Psi_{\ell_{3}}\left(\theta_{2}\right)\left|u^{-1}\right| \Psi_{\ell_{2}}\left(\theta_{1}\right) \Psi_{\ell_{4}}\left(\theta_{2}\right)\right\rangle .
$$

Using the partial-wave expansion (8) and the relation

$$
\begin{aligned}
\left\langle\ell_{1} \ell_{2} \ell_{3}\right\rangle & =\int Y_{\ell_{1}}(\theta) Y_{\ell_{2}}(\theta) Y_{\ell_{3}}(\theta) \sin \theta d \theta \\
& =\sqrt{\frac{\left(2 \ell_{1}+1\right)\left(2 \ell_{2}+1\right)\left(2 \ell_{3}+1\right)}{4 \pi}}\left(\begin{array}{ccc}
\ell_{1} & \ell_{2} & \ell_{3} \\
0 & 0 & 0
\end{array}\right)^{2}
\end{aligned}
$$

between the integrals of three spherical harmonics and the Wigner $3 j$-symbols [33], we find

$$
G_{\ell_{1} \ell_{2}}^{\ell_{3} \ell_{4}}=\frac{(-1)^{\ell_{3}+\ell_{4}}}{R} \sum_{\ell=0}^{\infty} \frac{4 \pi}{2 \ell+1}\left\langle\ell_{1} \ell_{2} \ell\right\rangle\left\langle\ell_{3} \ell_{4} \ell\right\rangle,
$$

where selection rules [33] restrict the terms in the sum.

The UHF energy is then given by

$$
E^{\mathrm{UHF}}=\sum_{\ell=0}^{\infty} C_{\ell}^{2} \frac{\ell(\ell+1)}{R^{2}}+\sum_{\ell_{1}, \ell_{2}=0}^{\infty} C_{\ell_{1}} C_{\ell_{2}} F_{\ell_{1} \ell_{2}} .
$$

The first term is the kinetic energy and is positive. However, for sufficiently large $R$, this is outweighed by negative contributions in the second term and it is these that drive the symmetry-breaking process.

For computational reasons, we truncate the sum in Eq. (15) at $\ell=L$ but, for all of the radii $R$ considered in this study, we found that $L=15$ suffices to obtain $E^{\mathrm{UHF}}$ with an accuracy of $10^{-12}$.

As Table I and Fig. 1 show, the UHF solution becomes lower than the RHF one for $R>R^{\text {crit }} \approx 1.5$ and the UHF, not RHF, energy behaves correctly for large $R$. Specifically, it can be shown that

$$
\begin{gathered}
\lim _{R \rightarrow \infty} R E^{\mathrm{RHF}}=1, \\
\lim _{R \rightarrow \infty} R E^{\mathrm{UHF}}=1 / 2 .
\end{gathered}
$$

The UHF result reflects the Coulomb interaction between two electrons localized on opposite sides of the sphere [17], a phenomenon known as Wigner crystallization $[10,11]$. The difference between the UHF and exact energies (i.e., the correlation energy) appears to decay as $O\left(R^{-3 / 2}\right)$. 
TABLE I. RHF, UHF, and exact energies for various $R$.

\begin{tabular}{cccr}
\hline \hline$R$ & \multicolumn{1}{c}{$E^{\text {RHF }}$} & $E^{\mathrm{UHF}}$ & \multicolumn{1}{c}{$E^{\text {exact }}$} \\
\hline 0.0001 & 10000 & 10000 & 9999.772600495 \\
0.001 & 1000 & 1000 & 999.772706409 \\
0.01 & 100 & 100 & 99.773761078 \\
0.1 & 10 & 10 & 9.783873673 \\
0.2 & 5 & 5 & 4.794237154 \\
0.5 & 2 & 2 & 1.820600768 \\
1 & 1 & 1 & 0.852781065 \\
2 & 0.500000 & 0.489551 & 0.391958796 \\
3 & 0.333333 & 0.304783 & 0.247897526 \\
4 & 0.250000 & 0.215864 & 0.179210308 \\
5 & 0.200000 & 0.165161 & 0.139470826 \\
10 & 0.100000 & 0.072829 & 0.064525123 \\
20 & 0.050000 & 0.032983 & 0.030271992 \\
50 & 0.020000 & 0.012006 & 0.011363694 \\
100 & 0.010000 & 0.005708105 & 0.005487412 \\
1000 & 0.001000 & 0.000522363 & 0.000515686 \\
\hline \hline
\end{tabular}

\section{EXPANSION FOR SMALL $R$}

In Møller-Plesset perturbation theory, the Hamiltonian of the system is partitioned as

$$
\hat{H}=\hat{H}_{0}+\hat{V}
$$

where $\hat{H}_{0}$ is the zeroth-order Hamiltonian and $\hat{V}$ is a perturbation operator and, in our case, we have

$$
\begin{gathered}
\hat{H}_{0}=\hat{T}, \\
\hat{V}=u^{-1} .
\end{gathered}
$$

The ground-state wave function and energy are expanded

$$
\begin{gathered}
\Phi=\Phi^{(0)}+\Phi^{(1)}+\Phi^{(2)}+\Phi^{(3)}+\ldots, \\
E=E^{(0)}+E^{(1)}+E^{(2)}+E^{(3)}+\ldots .
\end{gathered}
$$

We will refer to $E^{(n)}$ as the $n$ th-order energy and define the $\mathrm{MP} n$ correlation energy as

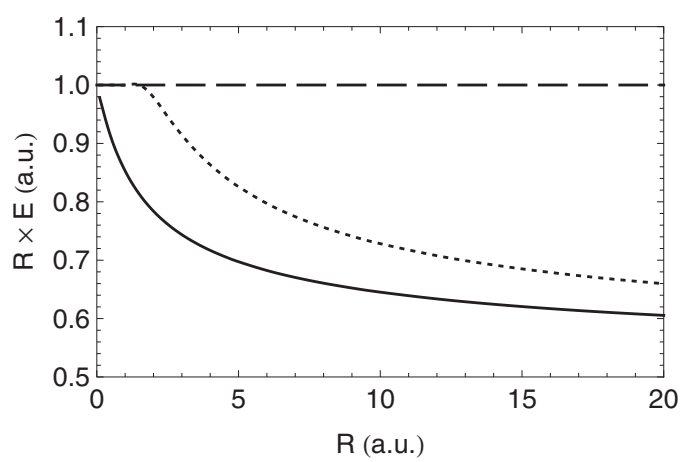

FIG. 1. $R \times E^{\mathrm{RHF}}$ (dashed), $R \times E^{\mathrm{UHF}}$ (dotted), and $R \times E^{\text {exact }}$ (solid) as a function of $R$.

$$
E^{\mathrm{MP} n}=\sum_{m=2}^{n} E^{(m)} .
$$

Dimensional analysis reveals that

$$
\begin{gathered}
\Phi=\frac{\phi_{0}}{R^{2}}+\frac{\phi_{1}}{R}+\phi_{2}+\phi_{3} R+\phi_{4} R^{2}+\phi_{5} R^{3}+\ldots, \\
E=\frac{\varepsilon_{0}}{R^{2}}+\frac{\varepsilon_{1}}{R}+\varepsilon_{2}+\varepsilon_{3} R+\varepsilon_{4} R^{2}+\varepsilon_{5} R^{3}+\ldots,
\end{gathered}
$$

where the $\phi_{n}$ are functions of $\theta$ and the $\varepsilon_{n}$ are numbers. From Eqs. (12) and (13), we see $\phi_{0}=1 / 4 \pi, \varepsilon_{0}=0$, and $\varepsilon_{1}$ $=1$.

The excited eigenfunctions of $\hat{H}_{0}$ are given by

$$
\Phi_{\ell_{1} m_{1}}^{\ell_{2} m_{2}}\left(\Omega_{1}, \Omega_{2}\right)=\Psi_{\ell_{1} m_{1}}\left(\Omega_{1}\right) \Psi_{\ell_{2} m_{2}}\left(\Omega_{2}\right),
$$

and we can expand the exact wave function $\Phi$ in this basis. However, for the ${ }^{1} S$ ground state, angular-momentum theory $[33,34]$ limits the combinations of $\ell_{1}, \ell_{2}, m_{1}$, and $m_{2}$ that contribute, and it is more efficient to expand $\Phi$ in the basis of two-electron functions

$$
\Phi_{\ell}(\theta)=\frac{\sqrt{2 \ell+1}}{4 \pi R^{2}} P_{\ell}(\cos \theta),
$$

which are eigenfunctions of $\hat{T}$ with eigenvalues

$$
E_{\ell}=\frac{\ell(\ell+1)}{R^{2}} .
$$

\section{A. First-order wave function}

In the intermediate normalization, the first-order wave function is

$$
\begin{aligned}
\Phi^{(1)}(\theta) & \equiv \frac{\phi_{1}(\theta)}{R} \\
& =\sum_{\ell=1}^{\infty} \frac{\left\langle\Phi^{\mathrm{RHF}}|\hat{V}| \Phi_{\ell}\right\rangle}{E_{0}-E_{\ell}} \Phi_{\ell}(\theta) \\
& =-\frac{1}{4 \pi R} \sum_{\ell=1}^{\infty} \frac{1}{\ell(\ell+1)} P_{\ell}(\cos \theta) .
\end{aligned}
$$

Using the Legendre generating function

$$
\sum_{\ell=0}^{\infty} P_{\ell}(x) t^{\ell}=\frac{1}{\sqrt{1-2 x t+t^{2}}},
$$

the sum in Eq. (34) can be found in closed-form, yielding

$$
\Phi^{(1)}(\theta)=\frac{1}{4 \pi R}\left[2 \ln \left(1+\sin \frac{\theta}{2}\right)-1\right],
$$

or, equivalently,

$$
\Phi^{(1)}(u)=\frac{1}{4 \pi R}\left[2 \ln \left(1+\frac{u}{2 R}\right)-1\right],
$$

and these yield the normalized first-order wave function 
TABLE II. Correlation energies (relative to UHF and multiplied by -1) from various models for various $R$.

\begin{tabular}{|c|c|c|c|c|c|c|c|}
\hline$R$ & MP2 & MP3 & MP4 & MP5 & Hylleraas & Seidl & Exact $^{\mathrm{a}}$ \\
\hline 0.0001 & 0.227411 & 0.227399504071 & 0.227399504574 & 0.227399504574 & 0.222212 & & 0.227399504574 \\
\hline 0.001 & 0.227411 & 0.227293541 & 0.227293591147 & 0.227293591133 & 0.222123 & & 0.227293591133 \\
\hline 0.01 & 0.227411 & 0.226234 & 0.226238936 & 0.226238922473 & 0.221237 & & 0.226238922463 \\
\hline 0.1 & 0.227411 & 0.215638 & 0.216140 & 0.216126387 & 0.212574 & $0.2175^{\mathrm{b}}$ & 0.216126326630 \\
\hline 0.2 & 0.227411 & 0.203864 & 0.205875 & 0.205763261 & 0.203406 & $0.2064^{\mathrm{b}}$ & 0.205762846030 \\
\hline 0.5 & 0.227411 & 0.168543 & 0.181112 & 0.179367 & 0.178908 & $0.1796^{\mathrm{b}}$ & 0.179399232168 \\
\hline \multirow[t]{2}{*}{1} & 0.227411 & 0.109674 & 0.159950 & 0.145992 & 0.147181 & $0.1473^{b}$ & 0.147218934944 \\
\hline & $e-e$ & LR0 & LR1 & LR2 & & & \\
\hline 2 & 0.239551 & 0.062774 & 0.094024 & 0.095405 & 0.096444 & $0.0977^{\mathrm{c}}$ & 0.097591955594 \\
\hline 3 & 0.138116 & 0.041890 & 0.055780 & 0.056281 & 0.054783 & & 0.056885070442 \\
\hline 4 & 0.090864 & 0.028352 & 0.036176 & 0.036420 & 0.033984 & & 0.036653426934 \\
\hline 5 & 0.065161 & 0.020440 & 0.025440 & 0.025580 & 0.022707 & $0.0257^{\mathrm{c}}$ & 0.025690364031 \\
\hline 10 & 0.022829 & 0.007018 & 0.008268 & 0.008292 & 0.005129 & $0.0083^{\mathrm{c}}$ & 0.008303955973 \\
\hline 20 & 0.007983 & 0.002393 & 0.002706 & 0.002710 & 0.000154 & & 0.002711198384 \\
\hline 50 & 0.002006 & 0.000592 & 0.000642054 & 0.000642496 & -0.000860 & & 0.000642573605 \\
\hline 100 & 0.000708 & 0.000187 & 0.000220605 & 0.000220683 & -0.000679 & & 0.000220692615 \\
\hline 1000 & 0.000022 & 0.000006552 & 0.000006677055 & 0.000006677302 & -0.000112 & & 0.000006677311 \\
\hline
\end{tabular}

${ }^{\mathrm{a}}$ From the polynomial wave function in Sec. VI D.

${ }^{\mathrm{b}}$ Native result of Ref. [17].

${ }^{\mathrm{c}}$ Correlation energy form Ref. [17] relative to the UHF energy.

$$
\Phi^{\mathrm{MP} 1}(\theta)=\frac{\Phi^{\mathrm{RHF}}+\Phi^{(1)}(\theta)}{\sqrt{1+(16 \ln 2-11) R^{2}}} .
$$

The true ground-state wave function must be nodeless. However, it is easy to show that the MP1 wave function possesses a node if $R>1$, leading us to anticipate that $\Phi^{\mathrm{MP} 1}$ will be a poor wave function for large spheres.

\section{B. Second- and third-order energies}

According to the Wigner $2 n+1$ rule [35], the first-order wave function generates the second- and third-order energies. The second-order energy, which has previously been found by Seidl [17], is given by

$$
E^{(2)} \equiv \varepsilon_{2}=\left\langle\Phi^{\mathrm{RHF}}|\hat{V}| \Phi^{(1)}\right\rangle=4 \mathcal{L}-3=-0.227411278 \ldots,
$$

where $\mathcal{L}=\ln 2$. As Table II shows, the MP2 correlation energy is an excellent approximation for small $R$ but, because it is independent of $R$, it is poor for large $R$.

It is surprising to find that $E^{(2)}$ is so much larger than the limiting correlation energies [36] of the helium-like ions $(-0.0467)$ or Hooke's law atoms $(-0.0497)$.

The third-order energy is given by

$$
E^{(3)} \equiv \varepsilon_{3} R=\left\langle\Phi^{(1)}\left|\hat{V}-E^{(1)}\right| \Phi^{(1)}\right\rangle,
$$

and this yields

$$
\varepsilon_{3}=8\left(\mathcal{L}^{2}-5 \mathcal{L}+3\right)=+0.117736889 \ldots,
$$

which agrees with Seidl's rough estimate [17]. Table II shows that MP3 gives an improvement over MP2 but that it, too, eventually breaks down as $R$ increases.

\section{Second-order wave function}

To find the fourth- and fifth-order energies, we need the second-order wave function. This is given by

$$
\Phi^{(2)}(\theta)=\sum_{\ell=1}^{\infty} \frac{\left\langle\Phi^{(1)}\left|\hat{V}-E^{(1)}\right| \Phi_{\ell}\right\rangle}{E_{0}-E_{\ell}} \Phi_{\ell}(\theta),
$$

which yields

$$
\begin{aligned}
\Phi^{(2)}(\theta)= & \frac{1}{4 \pi} \sum_{\ell_{1}, \ell_{2}=1}^{\infty} \sum_{\ell=\left|\ell_{1}-\ell_{2}\right|}^{\ell_{1}+\ell_{2}} \frac{2 \ell_{2}+1}{\ell_{1}\left(\ell_{1}+1\right) \ell_{2}\left(\ell_{2}+1\right)} \\
& \times\left(\begin{array}{ccc}
\ell_{1} & \ell_{2} & \ell \\
0 & 0 & 0
\end{array}\right)^{2} P_{\ell_{2}}(\cos \theta) .
\end{aligned}
$$

Using the identity

$$
\sum_{\ell=1}^{\infty} \frac{2 \ell+1}{\ell(\ell+1)} P_{\ell}(x) P_{\ell}(y)=-\ln \frac{(1-x)(1+y)}{4}-1,
$$

for $x \leq y$, we eventually obtain 


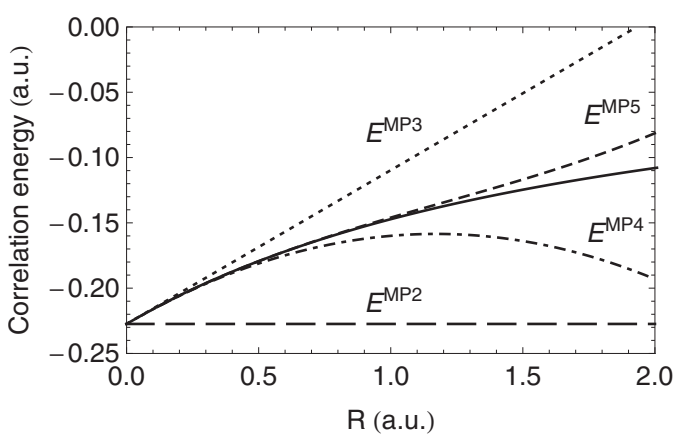

FIG. 2. MP $n$ correlation energies as a function of $R$. The exact correlation energy is shown as the solid curve.

$$
\begin{aligned}
\Phi^{(2)}(u)= & \left(2 \mathcal{L}^{2}-2 \mathcal{L}+5\right) \phi_{0}+(2 \mathcal{L}-5) \phi_{1}(u)-\frac{\pi}{4} \\
& +\frac{1}{\pi}\left[L i_{2}\left(\frac{1}{2}-\frac{u}{4 R}\right)-2 L i_{2}\left(-\frac{u}{2 R}\right)\right],
\end{aligned}
$$

where $L i_{2}$ is the dilogarithm function [37].

\section{Fourth- and fifth-order energies}

The Wigner $2 n+1$ rule and the closed-form expression of $\Phi^{(2)}$ yield the fourth- and fifth-order coefficients

$$
\begin{aligned}
\varepsilon_{4}= & \frac{16}{3}\left(4 \mathcal{L}^{3}-42 \mathcal{L}^{2}+96 \mathcal{L}-45\right)-12 \zeta(3) \\
= & -0.050275600 \ldots, \\
\varepsilon_{5}= & \frac{32}{3}\left(5 \mathcal{L}^{4}-90 \mathcal{L}^{3}+450 \mathcal{L}^{2}-660 \mathcal{L}+252\right) \\
& +(216-80 \mathcal{L}) \zeta(3) \\
= & +0.013957832 \ldots,
\end{aligned}
$$

where $\zeta$ is the Riemann zeta function.

The MPn correlation energies for various values of $R$ are reported in Table II and illustrated in Fig. 2. The results show that MP4 and MP5 are very accurate for small $R$ and, indeed, the latter is reasonable up to $R \approx 1$.

The MP expansion converges for radii $R$ within the radius of convergence

$$
R^{\mathrm{cvg}}=\lim _{n \rightarrow \infty}\left|\frac{\varepsilon_{n}}{\varepsilon_{n+1}}\right| .
$$

From our results, it seems that $R^{\mathrm{cvg}}>2$ but it is not possible to be more precise than this $[17,18]$.

\section{EXPANSION FOR LARGE R}

\section{A. Harmonic approximation}

For LR, the potential dominates the kinetic energy and the electrons tend to localize on opposite sides of the sphere. The classical mechanical energy would be

$$
E^{e-e}=\frac{1}{2 R}
$$

but, quantum mechanically, the kinetic energies of the electrons cannot vanish and each electron therefore maintains a zero-point oscillation around its equilibrium position with an angular frequency $\omega$. Such phenomena are ubiquitous in strongly correlated systems, as demonstrated by Seidl and his co-workers $[17,18,38-40]$.

In this limit, the supplementary angle $\xi=\pi-\theta$ is the natural coordinate and the Hamiltonian becomes

$$
\hat{H}=-\frac{1}{R^{2}}\left(\frac{d^{2}}{d \xi^{2}}+\cot \xi \frac{d}{d \xi}\right)+\frac{1}{2 R} \sec \frac{\xi}{2} .
$$

For small oscillations $(\xi \simeq 0)$, the Taylor series,

$$
\begin{gathered}
\cot \xi=\xi^{-1}-\xi / 3-\xi^{3} / 45+\ldots, \\
\sec (\xi / 2)=1+\xi^{2} / 8+5 \xi^{4} / 384+61 \xi^{6} / 46080+\ldots,
\end{gathered}
$$

yield the harmonic-oscillator Hamiltonian

$$
\hat{H}^{\omega}=-\frac{1}{R^{2}}\left(\frac{d^{2}}{d \xi^{2}}+\frac{1}{\xi} \frac{d}{d \xi}\right)+\frac{1}{2 R}\left(1+\frac{\xi^{2}}{8}\right),
$$

whose ground-state wave function and energy are

$$
\begin{gathered}
\Phi_{0}^{\omega}(\xi)=\frac{1}{2 \sqrt{2} \pi R^{7 / 4}} \exp \left(-\sqrt{R} \xi^{2} / 8\right), \\
E^{\mathrm{LR} 0} \equiv \mathcal{E}^{(0)}=\frac{1}{2 R}+\frac{1}{2 R^{3 / 2}} .
\end{gathered}
$$

The second term is the zero-point energy associated with harmonic oscillations of angular frequency $\omega=1 / R^{3 / 2}$ and it appears that this is the leading error in the UHF description at large $R$.

\section{B. First and second anharmonic corrections}

By analogy with the small- $R$ expansion (30), we would like to construct a large- $R$ asymptotic expansion

$$
E \sim \mathcal{E}^{(0)}+\mathcal{E}^{(1)}+\mathcal{E}^{(2)}+\ldots=\frac{\eta_{1}}{R}+\frac{\eta_{2}}{R^{3 / 2}}+\frac{\eta_{3}}{R^{2}}+\frac{\eta_{4}}{R^{5 / 2}}+\ldots
$$

where we know $\eta_{1}=\eta_{2}=1 / 2$. The $n$th excited state of the Hamiltonian (53) has the wave function and energy

$$
\begin{gathered}
\Phi_{n}^{\omega}(\xi)=L_{n}\left(\sqrt{R} \xi^{2} / 4\right) \Phi_{0}^{\omega}(\xi), \\
E_{n}^{\omega}=\left(n+\frac{1}{2}\right) \omega,
\end{gathered}
$$

where $L_{n}$ is the Laguerre polynomial of degree $n$ [29]. The anharmonic corrections, $\mathcal{E}^{(1)}$ and $\mathcal{E}^{(2)}$, can be found [41] using the perturbation operators

$$
\hat{W}^{(1)}=-\frac{1}{R^{2}} \frac{\xi}{3} \frac{d}{d \xi}+\frac{1}{2 R} \frac{5 \xi^{4}}{384},
$$




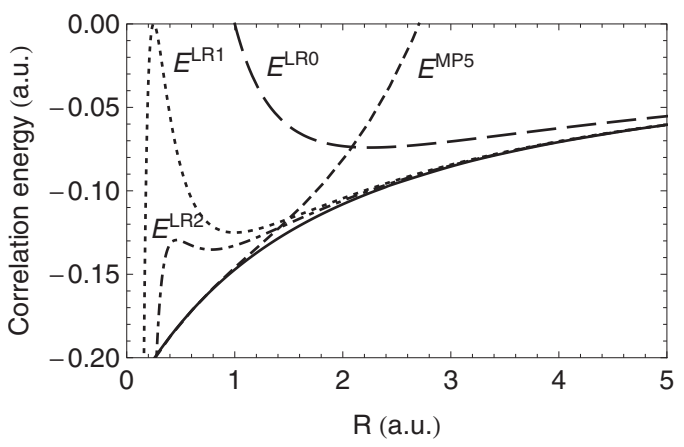

FIG. 3. Correlation energies (relative to RHF) from $E^{\mathrm{LR} 0}$ (dashed), $E^{\mathrm{LR} 1}$ (dotted), and $E^{\mathrm{LR} 2}$ (dot-dashed), and $E^{\mathrm{MP} 5}$ (small dash) and $E^{\text {exact }}$ (solid) as a function of $R$.

$$
\hat{W}^{(2)}=-\frac{1}{R^{2}} \frac{\xi^{3}}{45} \frac{d}{d \xi}+\frac{1}{2 R} \frac{61 \xi^{6}}{46080} .
$$

The first-order correction is

$$
\mathcal{E}^{(1)}=\left\langle\Phi_{0}^{\omega}\left|\hat{W}^{(1)}\right| \Phi_{0}^{\omega}\right\rangle=4 \pi^{2} R^{4} \int_{0}^{\infty} \Phi_{0}^{\omega}(\xi) \hat{W}^{(1)} \Phi_{0}^{\omega}(\xi) \xi d \xi,
$$

and this yields $\eta_{3}=-1 / 8$ and therefore

$$
E^{\mathrm{LR} 1}=\frac{1}{2 R}+\frac{1}{2 R^{3 / 2}}-\frac{1}{8 R^{2}} .
$$

The second-order correction is

$$
\mathcal{E}^{(2)}=\sum_{n=1}^{\infty} \frac{\left\langle\Phi_{0}^{\omega}\left|\hat{W}^{(1)}\right| \Phi_{n}^{\omega}\right\rangle^{2}}{E_{0}^{\omega}-E_{n}^{\omega}}+\left\langle\Phi_{0}^{\omega}\left|\hat{W}^{(2)}\right| \Phi_{0}^{\omega}\right\rangle,
$$

but because of the orthogonality and recurrence relations of Laguerre polynomials [29], only the first two terms in the sum in Eq. (63) are nonzero and one finds $\eta_{4}=-1 / 128$, and therefore

$$
E^{\mathrm{LR} 2}=\frac{1}{2 R}+\frac{1}{2 R^{3 / 2}}-\frac{1}{8 R^{2}}-\frac{1}{128 R^{5 / 2}} .
$$

From the results in Table II and Fig. 3, it seems that the asymptotic expansion converges toward the exact energy and is reasonably accurate for $R>3$.

Through judicious use of the fifth-order truncation of Eq. (30) and the second-order truncation of Eq. (56), one can predict satisfactory energies over a wide range of $R$ values. However, there remains a region $(1 \leq R \leq 3)$ where both the small- $R$ and large- $R$ solutions are inadequate.

\section{VARIATIONAL WAVE FUNCTIONS}

\section{A. Configuration interaction}

We begin with a configuration-interaction (CI) treatment wherein the wave function is expanded as
TABLE III. Convergence of correlation energies with respect to the number $L$ of terms in the CI, R12-CI, and Hylleraas wave functions. All results pertain to the sphere with $R=1$.

\begin{tabular}{rccc}
\hline \hline \multicolumn{1}{c}{ CI } & R12-CI & Polynomial \\
\hline 0 & -0.000000 & -0.147180860 & -0.000000000000 \\
1 & -0.131665 & -0.147185454 & -0.147180859845 \\
2 & -0.141241 & -0.147202916 & -0.147218627134 \\
3 & -0.144065 & -0.147209904 & -0.147218930072 \\
4 & -0.145273 & -0.147213200 & -0.147218934845 \\
5 & -0.145900 & -0.147214987 & -0.147218935941 \\
10 & -0.146847 & -0.147217796 & -0.147218935944 \\
15 & -0.147047 & -0.147218405 & -0.147218935944 \\
20 & -0.147120 & -0.147218631 & -0.147218935944 \\
25 & -0.147155 & -0.147218738 & -0.147218935944 \\
30 & -0.147174 & -0.147218797 & -0.147218935944 \\
35 & -0.147186 & -0.147218833 & -0.147218935944 \\
40 & -0.147194 & -0.147218257 & -0.147218935944 \\
\hline \hline
\end{tabular}

$$
\Phi_{L}^{\mathrm{CI}}(\theta)=\sum_{\ell=0}^{L} T_{\ell} \Phi_{\ell}(\theta)
$$

in the Legendre polynomial basis set (32). The resulting energy $E_{L}^{\mathrm{CI}}$ is the lowest eigenvalue of the CI matrix

$$
\left\langle\Phi_{\ell_{1}}|\hat{H}| \Phi_{\ell_{2}}\right\rangle=\frac{\ell_{1}\left(\ell_{1}+1\right)}{R^{2}} \delta_{\ell_{1}, \ell_{2}}+\frac{1}{R_{\ell=\left|\ell_{1}-\ell_{2}\right|}} \sum_{\frac{\ell_{1}+\ell_{2}}{2 \ell+1}}^{\ell_{1}}\left\langle\ell_{1} \ell_{2} \ell\right\rangle,
$$

where $\left\langle\ell_{1} \ell_{2} \ell\right\rangle$ is defined by Eq. (18).

The CI energy as the maximum angular momentum $L$ increases is reported in Table III. It converges very slowly and even $L=40$ yields an accuracy of only $10^{-4}$. The reason for this slow convergence-the failure of Eq. (65) to satisfy the Kato cusp condition-is well known.

\section{B. Hylleraas}

The simplest possible wave function with a cusp is

$$
\Phi^{\mathrm{Hy}}=1+\gamma u,
$$

which has an explicit linear dependence on the interelectronic distance $u$. Kato proved [25] that $\gamma=1 / 2$ in normal singlet states but, because our electrons are confined to a sphere, this $\gamma$ does not apply (see below).

Using the partial-wave expansion

$$
u=R \sum_{\ell=0}^{\infty}\left(\frac{1}{2 \ell+3}-\frac{1}{2 \ell-1}\right) P_{\ell}(\cos \theta),
$$

one finds that the energy is

$$
E^{\mathrm{Hy}}(\gamma)=\frac{6+3 \gamma(\gamma+4) R+8 \gamma^{2} R^{2}}{2 R\left(3+8 \gamma R+6 \gamma^{2} R^{2}\right)},
$$

and minimizing this with respect to $\gamma$ yields 


$$
\begin{aligned}
\gamma^{\mathrm{opt}} & =\frac{12}{9-12 R+\sqrt{81+72 R+48 R^{2}}}, \\
E^{\mathrm{Hy}} & =\frac{9+12 R-\sqrt{81+72 R+48 R^{2}}}{8 R^{2}} .
\end{aligned}
$$

Correlation energies obtained from Eq. (71) for several values of $R$ are reported in Table II. Despite the simplicity of the wave function, its energies are surprisingly good with a maximum deviation of 0.003 for large $R$ and 0.005 for small $R$. As $R$ tends to zero, the correlation energy approaches -0.222222 , which is close to the exact value -0.227411 . However, as $R$ becomes large, one can show that $E^{\mathrm{Hy}}$ $\sim 1 /(1.58 R)$, which lies between the RHF and UHF energies. The Hylleraas wave function is thus a useful alternative to the small- and large- $R$ solutions in the problematic intermediate region $(1 \lesssim R \leqq 3)$ with errors of $0.000,0.0011$, and 0.0021 for $R=1,2$, and 3 , respectively.

\section{R12-CI}

Using Hylleraas wave function (67) as the reference for a CI expansion yields the R12-CI wave function

$$
\Phi_{L}^{R 12-\mathrm{CI}}(\theta)=\hat{P} \Phi^{\mathrm{Hy}}(\theta)+\sum_{\ell=1}^{L} T_{\ell} \Phi_{\ell}(\theta),
$$

where

$$
\hat{P}=\hat{I}-\sum_{\ell=1}^{\infty}\left|\Phi_{\ell}\right\rangle\left\langle\Phi_{\ell}\right|
$$

is a projection operator that ensures orthogonality between the reference wave function and the excited determinants, and $\hat{I}$ is the identity operator. The coupling coefficients between two basis functions are the same as those for the conventional CI calculation [Eq. (66)] but with a correction for the matrix element,

$$
\left\langle\Phi^{\mathrm{Hy}}|\hat{H}| \Phi_{\ell}\right\rangle=\left\langle\Phi^{\mathrm{RHF}}|\hat{H}| \Phi_{\ell}\right\rangle+\gamma \frac{\ell(\ell+1)}{R \sqrt{2 \ell+1}}\left(\frac{1}{2 \ell+3}-\frac{1}{2 \ell-1}\right),
$$

involving the ground state and the excited determinants. It is no longer possible to optimize $\gamma$ in closed form so we used the value given by Eq. (70).

As Table III shows, the R12-CI energies converge much more rapidly with $L$ than the CI energies and, for example, $E_{2}^{R 12-\mathrm{CI}}$ is more accurate than $E_{40}^{\mathrm{CI}}$. This illustrates the importance of including a term that is linear in $u$. However, although this term enhances the initial convergence rate, the asymptotic behavior of the CI and R12-CI schemes are identical. Therefore, we now investigate the effect of including higher-order $u$ terms.

\section{Polynomial}

In terms of the distance $u$, the Hamiltonian is

$$
\hat{H}=\left(\frac{u^{2}}{4 R^{2}}-1\right) \frac{d^{2}}{d u^{2}}+\left(\frac{3 u}{4 R^{2}}-\frac{1}{u}\right) \frac{d}{d u}+\frac{1}{u},
$$

and a Kato-like analysis [25] reveals the cusp condition

$$
\frac{\Phi^{\prime}(0)}{\Phi(0)}=1
$$

which deviates from the normal value of $1 / 2$ [17].

The natural generalization of Hylleraas wave function (67) is a polynomial and it is convenient to select the orthonormal basis of Jacobi polynomials [29]

$$
\Xi_{\ell}(u)=\frac{\sqrt{\ell+1}}{4 \pi R^{2}} P_{\ell}^{(1,0)}\left(1-\frac{u}{R}\right),
$$

and write the wave function as

$$
\Phi_{L}^{\text {poly }}=\sum_{\ell=0}^{L} c_{\ell} \Xi_{\ell}(u) \text {. }
$$

The energy $E_{L}^{\text {poly }}$ is the lowest eigenvalue of the matrix

$$
\left\langle\Xi_{i}|\hat{H}| \Xi_{j}\right\rangle=\frac{\left(m^{2}-1\right)\left(\alpha m+\delta_{i, j}\right)}{4 R^{2}}+\frac{\alpha m}{R},
$$

where $m=\min (i, j)$ and $\alpha=\sqrt{\frac{\min (i, j)}{\max (i, j)}}$.

Table III reveals the remarkable convergence of $E_{L}^{\text {poly }}$. Using $L=40$ and $R=1$, for example, we find

$$
\begin{aligned}
E_{40}^{\text {poly }}= & 0.852781065056462665400437 \\
& 966038710264283589518406 \\
& 360162484313983 .
\end{aligned}
$$

The convergence is slower for larger values of $R$ but still impressive. For example, using $L=40$ and $R=1000$, the energy is still correct to 49 digits. The ease with which we can obtain these Schrödinger eigenvalues can be traced to the fact that the polynomial basis efficiently models all of the singularities (the first-order cusp, the third-order cusp, etc.) in the exact wave function.

In recent work [17], Seidl reported correlation energies based on his numerical integration of the Schrödinger equation from $\theta=0$ to $\pi$ using Eq. (4) and we have included these in Table II. It appears that some of his energies for small $R$ are slightly inaccurate.

\section{CONCLUSION}

In this paper, we have reported results for the ground state of a simple two- electron system that is described by a single parameter $R$. Although we cannot solve its Schrödinger equation in closed form, we have found accurate wave functions and energies for small $R$ (the weakly correlated limit) and large $R$ (the strongly correlated limit). For $R \ll 1$, MøllerPlesset perturbation theory yields results close to the exact solution; for $R \gg 1$, accurate results can be found by considering the zero-point oscillations of the appropriate Wigner molecule. 
We have also explored variational schemes that yield satisfactory results for all $R$. In particular, we have discovered a polynomial wave function that easily yields results of any required accuracy.

We believe that our results will be useful in the future development of accurate correlation functionals within density-functional theory $[42,43]$ and intracule functional theory [44-49].

\section{ACKNOWLEDGMENTS}

P.M.W.G. thanks the APAC Merit Allocation Scheme for a grant of supercomputer time and the Australian Research Council (Grant No. DP0664466) for funding. We also thank Yves Bernard for helpful comments on the paper, and fruitful discussions.
[1] P. Hohenberg and W. Kohn, Phys. Rev. 136, B864 (1964).

[2] W. Kohn and L. Sham, Phys. Rev. 140, A1133 (1965).

[3] R. G. Parr and W. Yang, Density Functional Theory for Atoms and Molecules (Oxford University Press, New York, 1989).

[4] N. R. Kestner and O. Sinanoglu, Phys. Rev. 128, 2687 (1962).

[5] S. Kais, D. R. Herschbach, and R. D. Levine, J. Chem. Phys. 91, 7791 (1989).

[6] M. Taut, Phys. Rev. A 48, 3561 (1993).

[7] A. Alavi, J. Chem. Phys. 113, 7735 (2000).

[8] D. C. Thompson and A. Alavi, Phys. Rev. B 66, 235118 (2002).

[9] D. C. Thompson and A. Alavi, J. Chem. Phys. 122, 124107 (2005).

[10] D. C. Thompson and A. Alavi, Phys. Rev. B 69, 201302(R) (2004).

[11] E. Wigner, Phys. Rev. 46, 1002 (1934).

[12] G. S. Ezra and R. S. Berry, Phys. Rev. A 25, 1513 (1982).

[13] G. S. Ezra and R. S. Berry, Phys. Rev. A 28, 1989 (1983).

[14] P. C. Ojha and R. S. Berry, Phys. Rev. A 36, 1575 (1987).

[15] R. J. Hinde and R. S. Berry, Phys. Rev. A 42, 2259 (1990).

[16] J. W. Warner and R. S. Berry, Nature (London) 313, 160 (1985).

[17] M. Seidl, Phys. Rev. A 75, 062506 (2007).

[18] M. Seidl, J. P. Perdew, and S. Kurth, Phys. Rev. Lett. 84, 5070 (2000).

[19] C. Møller and M. S. Plesset, Phys. Rev. 46, 618 (1934).

[20] E. A. Hylleraas, Adv. Quantum Chem. 1, 1 (1964).

[21] W. Kutzelnigg, Theor. Chim. Acta 68, 445 (1985).

[22] W. Klopper and W. Kutzelnigg, Chem. Phys. Lett. 134, 17 (1987).

[23] W. Klopper and W. Kutzelnigg, J. Phys. Chem. 94, 5625 (1990).

[24] W. Kutzelnigg and W. Klopper, J. Chem. Phys. 94, 1985 (1991).

[25] T. Kato, Commun. Pure Appl. Math. 10, 151 (1957).

[26] R. T. Pack and W. Byers Brown, J. Chem. Phys. 45, 556 (1966).

[27] A. Szabo and N. S. Ostlund, Modern Quantum Chemistry: Introduction to Advanced Structure Theory (Dover publications Inc., Mineola, NY, 1989).
[28] G. B. Arfken, Mathematical Methods for Physicists (Academic Press, New York, 1966).

[29] M. Abramowitz and I. A. Stegun, Handbook of Mathematical Functions with Formulas, Graphs and Mathematical Tables (Dover publications Inc., New York, 1972).

[30] J. Čížek and J. Paldus, J. Chem. Phys. 47, 3976 (1967).

[31] L. Paldus and J. Č́ízek, J. Chem. Phys. 52, 2919 (1970).

[32] R. Seeger and J. Pople, J. Chem. Phys. 66, 3045 (1977).

[33] A. R. Edmonds, Angular Momentum in Quantum Mechanics (Princeton University Press, Princeton, NJ, 1957).

[34] J. C. Slater, in Quantum Theory of Atomic Structures, International Series in Pure and Applied Physics (McGraw-Hill Book Company, Inc., New York, 1960), Vol. 2.

[35] T. Helgaker, P. Jørgensen, and J. Olsen, Molecular ElectronicStructure Theory (John Wiley \& Sons, Ltd., New York, 2000).

[36] P. M. W. Gill and D. P. O’Neill, J. Chem. Phys. 122, 094110 (2005).

[37] L. Lewin, Dilogarithms and Associated Functions (Macdonald, London, 1958).

[38] M. Seidl, J. P. Perdew, and M. Levy, Phys. Rev. A 59, 51 (1999).

[39] M. Seidl, Phys. Rev. A 60, 4387 (1999).

[40] M. Seidl, P. Gori-Giorgi, and A. Savin, Phys. Rev. A 75, 042511 (2007).

[41] C. M. Bender and T. T. Wu, Phys. Rev. 184, 1231 (1969).

[42] J. Sun, J. Chem. Theory Comput. 5, 708 (2009).

[43] P. Gori-Giorgi, G. Vignale, and M. Seidl, J. Chem. Theory Comput. 5, 743 (2009).

[44] P. M. W. Gill, D. L. Crittenden, D. P. O’Neill, and N. A. Besley, Phys. Chem. Chem. Phys. 8, 15 (2006).

[45] E. E. Dumont, D. L. Crittenden, and P. M. W. Gill, Phys. Chem. Chem. Phys. 9, 5340 (2007).

[46] D. L. Crittenden and P. M. W. Gill, J. Chem. Phys. 127, 014101 (2007).

[47] D. L. Crittenden, E. E. Dumont, and P. M. W. Gill, J. Chem. Phys. 127, 141103 (2007).

[48] Y. A. Bernard, D. L. Crittenden, and P. M. W. Gill, Phys. Chem. Chem. Phys. 10, 3447 (2008).

[49] J. K. Pearson, D. L. Crittenden, and P. M. W. Gill, J. Chem. Phys. 130, 164110 (2009). 